\title{
Expectational Business Cycles
}

\author{
Eran A. Guse* \\ Bank of Finland
}

July 30, 2004

\begin{abstract}
I introduce Expectational Business Cycles where aggregate activity fluctuates due to learning, heterogeneous updating rules and random changes in the social norm predictor. Agents use one of two updating rules to learn the equilibrium values while heterogeneity is dictated via an evolutionary process. Uncertainty of a new equilibrium, due to a shock to the structure of the economy, results in a sudden decrease in output. As agents learn the equilibrium, output slowly increases to its equilibrium value. These business cycles arrive faster, are longer and more severe as agents possess less rationality.
\end{abstract}

Key Words: Adaptive Learning; Aggregate Fluctuations; Heterogeneous Expectations; Multiple Equilibria; Rational Expectations

JEL Classification: C62, D84, E37

\section{Introduction}

Uncertainty affects the daily decisions we make in our lives for if we knew all our future lifetime events, we would certainly change our behavior in order to maximize actual total lifetime utility. Unfortunately, we are uncertain about events that affect our lives, the world and the economy

*Address correspondence to: Bank of Finland, P.O. Box 160, FIN-00101 Helsinki, Finland. Phone: +3589 183 2372. E-mail: eran.guse@bof.fi. This paper was written when I was a postdoctoral fellow at the Research Unit on Economic Structures and Growth (RUESG), Department of Economics, University of Helsinki. Financial support from the Academy of Finland, Yrjö Jahnsson Foundation, Bank of Finland and Nokia Group is gratefully acknowledged. I would like to thank Shankha Chakraborty, George Evans, Seppo Honkapohja, Van Kolpin, Sunny M.C. Wong, and the participants of the Bank of Finland Research Department seminars for useful comments and suggestions. All errors are mine. 
in both the micro and macro levels. This uncertainty affects each individual's behavior of consumption, investment, and employment. Better expectation mechanisms can lead to smaller forecasting mistakes and less uncertainty and thus lead to higher current and future benefits.

It is common knowledge among economists that there is more uncertainty in a recession that there is during an expansion. The median forecast error and the dispersion of GDP forecasts in the Survey of Professional Forecasters tend to increase during a recession. In a period of higher uncertainty, agents may be confused about whether there is a permanent or transitory shock to their income and well being. As a result, agents may be unsure how to smooth consumption and thus decrease it more than they would with greater certainty. This is demonstrated in Cogley (2001) where consumption is more volatile during a recession. Furthermore, firms may also be less certain about returns to investments during a recession. Potter (1999) finds that investors may take a stand of "wait and see" during times of uncertainty, thus decreasing investment.

There has been several different types of literature that have focused on the importance of learning as an endogenous propagation mechanism for the business cycle. The first type of models, I call the "Good-Bad" models, generate a business cycle from a Markov process of good and bad times. These models focus on providing explanation of the well known fact that the average business cycle is asymmetric where the arrival of the recession is quite prompt and the recovery is more drawn out. In Chalkley and Lee (1998), agents learn from their predecessors on the state of the economy with some noise. These agents decide whether to put in high or low effort based on their knowledge. If agents believe that they are in a "bad" state and see an aggregate increase, they may believe that the increase was from a stochastic shock and not a shift to the "good" state. Therefore, agents will adjust quickly in the bad state, but, due to risk aversion, the agents will slowly adjust in the "good" state. In González (1997), agents learn from others in good times creating informational economies of scale. During bad times, agents focus on their microeconomic activity rather than learning about macroeconomic activity. When there is a shock to the "bad" state, agents see this shock due to the informational economies of scale and react to the shock quickly. When the shock to the "good" state occurs, agents are unaware of this shock due to the loss of the informational economies of scale. The author suggests that in order for others to believe that they are back in the "good" state, some agents must experiment which could further increase aggregate activity. Finally, Nieuwerburgh and Veldcamp (2003) 
consider an RBC-like model with a Markov technology shock and informational economies of scale through production. They conclude that low production creates noisy estimates of recovery leading to a slower recovery.

The second type of models in the literature are those of learning or changes in expectations producing the business cycle. Farmer and Guo (1994) investigate a model with an aggregate technology that is subject to increasing returns. They demonstrate that the model can display fluctuations at business cycle frequencies due to sunspots even when there are no shocks to the fundamentals of the economy. Evans, Honkapohja, and Romer (1998) consider a model with multiple equilibria and produce a business cycle from a change in expectations via a Markov process. ${ }^{1}$ They find that the equilibrium of "growth cycles" is stable under a simple learning rule. Kasa (1995) considers a model where firms forecast the forecasts of other firms. He discovers that forecast errors can make a significant contribution to the propagation of business cycles.

Finally, the third type of literature is including learning in RBC models. Williams (2003a) finds that learning does not substantially change the volatility and the persistence of key economic variables. However, when agents learn about the structural features of the economy, there are much greater effects to volatility and persistence. This suggests that a less rational form of learning may work as a stronger propagation mechanism for the business cycle.

In this paper, I examine a model in which a Markov process or technology shocks do not directly create a business cycle. Following Williams (2003a), there is a less rational type of learning mechanism produced from heterogeneous learning mechanisms. I use the model discussed in Guse (2003b) and define a utility function based on uncertainty to describe the cyclical fluctuations. In this case, learning and changing learning behavior acts as a propagation mechanism of the business cycle. Furthermore, this model gives the same results of asymmetry in the business cycle as discussed in the literature.

\footnotetext{
${ }^{1}$ Farmer and Guo (1994) and Evans, Honkapohja, and Romer (1998) have the same basic idea: a non-convex economy that leads to stationary sunspot equilibria (SSE). In Farmer and Guo, there are SSE's near a single steady state, while in Evans, Honkapohja and Romer there are multiple steady states each with SSE's near them.
} 


\section{The Validity of Adaptive Learning}

One of the main conclusions of the adaptive learning literature is that a rational expectations equilibrium (REE) is relevant only when agents can learn the solution when their initial beliefs are off the equilibrium path. If the equilibrium is not learnable, then one should not consider it a possible solution when studying economic policy as an alternative result will occur when off the equilibrium path. If a REE is stable under learning, then when agents continue to learn the equilibrium using least squares or a closely related algorithm, ceterus peribus, the agents will learn the REE.

One objection to adaptive learning is, "why have we yet not learned the (stable) rational expectations equilibrium?" Those who feel this way must believe that the economy is static and forget that the world is always changing. Over the past thirty plus years, we have seen many structural changes to the world economy from an oil crisis to the development of the internet to the post September 11, 2001 economy. If people continued to form expectations in the same manner over these years, would this not be considered naive? This paper demonstrates an important fact that some economists maybe ignoring; people will change the way they form expectations due to "expectational shocks" to the economy.

In Guse (2003b), agents could differ in the way they formed expectations in the short run. Under certain situations, some agents using the "inefficient" predictor decided to switch to the "efficient" predictor. This process continued until all of the agents were using the same efficient predictor creating a "social norm" for forming expectations. This paper will show how a business cycle may occur due to a change in a "social norm" for forming expectations. Suppose that the economy is experiencing a period of expansion and there is a shock to the structure of the economy. This shock may be a change in preferences, monetary or fiscal policy, technology, or a major event that effects the world economy. From this shock, there may be another predictor that is now more efficient than the current "social norm" predictor. With agents now using a relatively inefficient predictor, there may be less investment and a decline in consumer confidence due to an increase in uncertainty. This may lead to a decline in growth and an increase in unemployment moving the economy into a recession. During this time, a small amount of agents may discover that the current predictor is inefficient and switch to using the 
new efficient predictor. Agents using the inefficient predictor may see this and decide to change to the other predictor while others will follow later. This process will lead to a new "social norm" for forming expectations and bring the economy into another expansion from agents now using an efficient predictor.

The goal of this paper is to introduce the concept of Expectational Business Cycles where aggregate fluctuations are produced from such a change in the social norm predictor. When this new predictor is introduced, agents slowly move to the efficient predictor and learn the new parameter values. Evolutionary dynamics and learning act as a propagation mechanism for the business cycle. When individuals learn, they tend to make large initial mistakes and then learn how to minimize these mistakes. As a result, learning is a prime candidate for an explanation of asymmetry in the business cycle.

\section{The Model}

The model discussed in this paper is a version of Taylor (1977). ${ }^{2}$ It is a self referential linear stochastic model with real balance effects consisting of four parts:

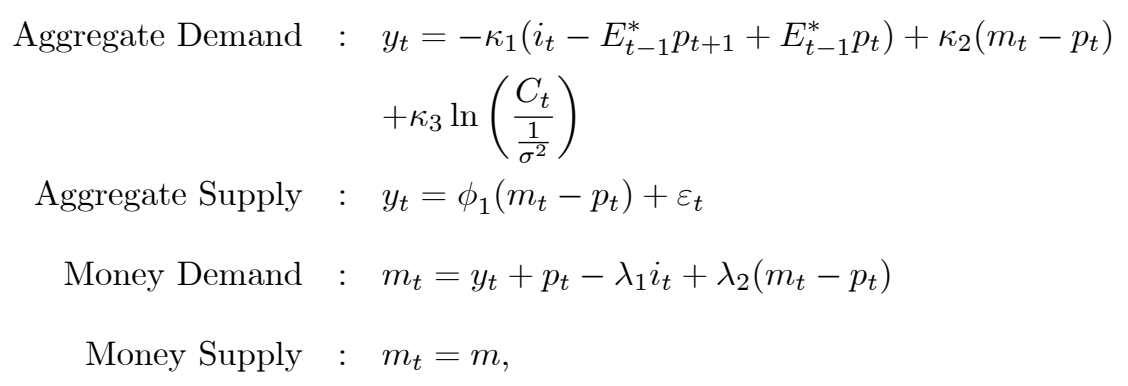

where $y_{t}$ is the logarithm of real output, $C_{t}$ is the current level of confidence, $i_{t}$ is the nominal interest rate, $p_{t}$ is the logarithm of the price of output, $m_{t}$ is the logarithm of the stock of nominal

\footnotetext{
${ }^{2}$ This model is not used in business cycle literature but is a current workhorse for the learning literature. I use this model as it is the simplest model with multiple stationary and learnable rational expectations equilibria. Future work will consider more commonly used models where multiple updating rules may be used.
} 
money balances, and $\varepsilon_{t}$ is a persistent random shock variable ${ }^{3}$

$$
\varepsilon_{t}=\rho \varepsilon_{t-1}+\xi_{t}
$$

where

$$
\xi_{t} \sim N\left(0, \sigma_{\xi}^{2}\right) .
$$

$\sigma^{2}$ represents the variance of the stochastic shock variable, $\tilde{\xi}_{t}$, defined below. All greek letters represent positive coefficients and $\lambda_{2} \leq 1 . E_{t-1}^{*}$ represents the not necessarily rational expectation operator at $t-1$.

This model now includes a confidence effect on real output that was not present in Taylor (1977). The confidence level is determined by how well the average agent can predict current and future prices. In this economy, agents will consume and invest more if they feel comfortable about their own predictions of the future. Therefore, the confidence coefficient can be motivated by precautionary savings by consumers and firms. Predictability will be determined by the average mean squared error (MSE). As the average MSE increases, the uncertainty of the future increases. Agents will choose not to consume and invest as much as before thus decreasing real GDP.

This model can be written in its reduced form as the following:

$$
p_{t}=\alpha_{t}+\beta_{0} E_{t-1}^{*} p_{t}+\beta_{1} E_{t-1}^{*} p_{t+1}+\rho v_{t-1}+\tilde{\xi}_{t}
$$

\footnotetext{
${ }^{3}$ The original Taylor model has separate shocks to aggregate demand and money demand as well as to aggregate supply. Including three shocks complicates the model, but does not change the results below.
} 
where $^{4}$

$$
\begin{aligned}
\alpha_{t} & =m-\frac{\beta_{0} \kappa_{3}}{\kappa_{1}} \ln \left(\frac{C_{t}}{\frac{1}{\sigma^{2}}}\right) \\
\beta_{0} & =-\frac{\lambda_{1} \kappa_{1}}{\lambda_{1}\left(\kappa_{2}-\phi_{1}\right)-\kappa_{1}\left(\phi_{1}-\left(1-\lambda_{2}\right)\right)} \\
\beta_{1} & =-\beta_{0} \\
v_{t} & =\beta_{0} *\left(\frac{\left(\lambda_{1}+\kappa_{1}\right)}{\lambda_{1} \kappa_{1}}\right) \varepsilon_{t} \\
\tilde{\xi}_{t} & =\beta_{0} *\left(\frac{\left(\lambda_{1}+\kappa_{1}\right)}{\lambda_{1} \kappa_{1}}\right) \xi_{t}
\end{aligned}
$$

and

$$
\tilde{\xi}_{t} \sim N\left(0, \sigma^{2}=\beta_{0}^{2} *\left(\frac{\left(\lambda_{1}+\kappa_{1}\right)}{\lambda_{1} \kappa_{1}}\right)^{2} \sigma_{\xi}^{2}\right) .
$$

With the inclusion of the confidence variable, the rational expectations equilibria does not change, so the MSE of each predictor and E-stability conditions are the same as in Guse (2003b).

\subsection{Choice of Predictors}

Assume that agents have the choice of using one of two predictors corresponding to two possible REE discussed below:

$$
\begin{array}{ll}
P L M_{1}: & p_{t}=a_{1}+\rho v_{t-1}+\tilde{\xi}_{t} \\
P L M_{2} & : \quad p_{t}=a_{2}+b_{2} p_{t-1}+\rho v_{t-1}+\tilde{\xi}_{t} .
\end{array}
$$

where agents recursively estimate the coefficients of their PLM to form expectations. If a proportion of $\mu$ agents uses $P L M_{1}$ and the remaining $(1-\mu)$ agents use $P L M_{2}$, then the actual law of motion (ALM) is:

$$
\begin{aligned}
p_{t}= & \alpha+\mu a_{1}\left(\beta_{0}+\beta_{1}\right)+(1-\mu) a_{2}\left(\beta_{0}+\beta_{1}\left(1+b_{2}\right)\right) \\
& +\left[(1-\mu) b_{2}\left(\beta_{0}+\beta_{1} b_{2}\right)\right] p_{t-1}+\rho v_{t-1}+\tilde{\xi}_{t}
\end{aligned}
$$

\footnotetext{
${ }^{4}$ The solutions for the reduced form in Taylor (1977) are incorrect, however, this does not affect his results.
} 
The above system defines a mapping from the PLM to the ALM as follows:

$$
T\left(\begin{array}{c}
a_{1} \\
a_{2} \\
b_{2}
\end{array}\right)=\left(\begin{array}{c}
\frac{\alpha+\mu a_{1}\left(\beta_{0}+\beta_{1}\right)+(1-\mu) a_{2}\left(\beta_{0}+\beta_{1}\left(1+b_{2}\right)\right)}{1-(1-\mu) b_{2}\left(\beta_{0}+\beta_{1} b_{2}\right)} \\
\alpha+\mu a_{1}\left(\beta_{0}+\beta_{1}\right)+(1-\mu) a_{2}\left(\beta_{0}+\beta_{1}\left(1+b_{2}\right)\right) \\
(1-\mu) b_{2}\left(\beta_{0}+\beta_{1} b_{2}\right)
\end{array}\right)
$$

The resulting equilibria are expressed as: ${ }^{5}$

$$
\begin{aligned}
a_{1} & =\frac{\alpha}{1-\beta_{0}-\beta_{1}} \\
a_{2} & =a_{1}\left(1-b_{2}\right) \\
b_{2} & =\frac{1-(1-\mu) \beta_{0}}{(1-\mu) \beta_{1}}
\end{aligned}
$$

or

$$
\begin{aligned}
& a_{1}=\frac{\alpha}{1-\beta_{0}-\beta_{1}} \\
& a_{2}=\frac{\alpha}{1-\beta_{0}-\beta_{1}} \\
& b_{2}=0
\end{aligned}
$$

Equilibrium (6) is referred to as the $\mathrm{AR}(1)$ mixed expectations equilibria (MEE). ${ }^{6}$ In this equilibrium, the proportion of agents using $P L M_{1}$ are underparameterizing the model when they are forming their expectations. $P L M_{2}$ will be referred to as the $\mathrm{AR}(1)$ predictor since these agents believe that the actual equilibrium will be equilibrium (6). Equilibrium (7) is referred to as the minimum state variable (MSV) MEE. Although the equilibria expectations in the MSV solution are homogenous, it will be considered heterogeneous expectations since two predictors are used to form expectations. $P L M_{1}$ will be referred to as the MSV predictor as these agents believe the true equilibrium is equilibrium (7).

Learnability of the two equilibria can be determined by the E-stability principle. ${ }^{7}$ Consider

\footnotetext{
${ }^{5}$ One restriction is that the $\mathrm{AR}(1) \mathrm{MEE}$ must be stationary for the first component of the T-map to be well-defined.

${ }^{6}$ Each equilibrium is referred to as "mixed" because it may be generated from two expectations predictors.

${ }^{7}$ For a detailed presentation of the E-stability Principle, see Evans and Honkapohja (2001).
} 
the following ordinary differential equation (ODE):

$$
\frac{d \phi}{d \tau}=T(\phi)-\phi
$$

where $\tau$ denotes notional, or artificial time. An equilibrium, or fixed point of the ODE, is E-stable if it is locally stable under the ODE. The following proposition, from Guse (2003a), presents the E-stability conditions for both equilibria under the above model.

Proposition 1: Assume that $\beta_{0}+\beta_{1}<1$. If $\beta_{0}<\frac{1}{1-\mu}$, then the MSV MEE is E-stable and the AR(1) MEE is E-unstable. If $\beta_{0}>\frac{1}{1-\mu}$, then the MSV MEE is E-unstable and the AR(1) MEE is E-stable.

One key result in Guse (2003a) is that the equilibria exchange stability at $\beta_{0}=\frac{1}{1-\mu}$ where the mean squared error of the MSV predictor under the AR(1) solution is minimized. This relationship will be important when considering predictor choice dynamics.

\subsection{Predictor Choice Dynamics}

Assume that there is a continuum agents where each agent's decision does not affect the state of the economy. Let $([0,1], \mathcal{B})$ be the underlying space where $[0,1]$ is the player set and $\mathcal{B}$ is the Borel subsets of $[0,1]$. Let $S_{i}=\left\{P L M_{1}, P L M_{2}\right\}$ be the set of strategies for each player $i$. Suppose that each player receives a payoff from choosing either strategy in the following manner:

$$
\begin{aligned}
v_{i}\left(s_{i}, \mu\right) & =\frac{1}{M S E_{1}}-K_{1}=U_{1} \text { if } S_{i}=P L M_{1} \\
& =\frac{1}{M S E_{2}}-K_{2}=U_{2} \text { if } S_{i}=P L M_{2}
\end{aligned}
$$

where $K_{j} \geq 0$ is the cost parameter for using the jth predictor discussed further below.

When considering predictor choice dynamics in such a model, one must consider when each solution is evolutionary E-stable. To theoretically evaluate an equilibrium for evolutionary Estability, I assume Fast-Slow dynamics which is a process where agents learn the corresponding parameter equilibria prior to each period when $\mu$ is updated using some form of a selection criterion. Therefore, the speed of parameter learning is infinitely faster than the speed of the 
population dynamics.

Definition 1: Assume that the model is updated using Fast-Slow dynamics. An MEE or REE, $\phi^{*}\left(\mu^{*}\right)$, is Evolutionary E-stable if for all $\mu \in[0,1]$ sufficiently close to $\mu^{*}(1) \mu_{t} \rightarrow \mu^{*}$ under the replicator dynamics and $(2) \phi\left(\mu_{t}\right)$ is E-stable for all $\mu_{t}$.

Here, $\phi(\mu)$ refers to an E-stable MEE that is determined by the level of heterogeneity, $\mu$, and $\phi^{*}\left(\mu^{*}\right)$ is the MEE determined by a Nash solution of $\mu^{*}$. Under evolutionary E-stability, if a mutation occurs to change the level of heterogeneity, then the system will return to the evolutionary E-stable MEE or REE. Furthermore, at each $\mu$ in the neighborhood of $\mu^{*}$, the corresponding MEE is E-stable. Like E-stability, this is a local condition, but unlike E-stability, the boundary of attraction may be determined under the replicator dynamics for each Nash solution.

The selection criterion used in this paper will be the replicator dynamics which is commonly used in evolutionary game theory. The replicator dynamics, in discrete time, is defined as follows:

$$
\mu_{t}=\left(\frac{\zeta+U_{1}}{\zeta+\mu_{t-1} * U_{1}+\left(1-\mu_{t-1}\right) * U_{2}}\right) * \mu_{t-1} .
$$

Equation (8) directs the population to use the more efficient predictor at time $t-1$. In the game, there is the possibility of convergence to homogeneous expectations due to the exponential nature of the replicator dynamics.

\subsection{Evolutionary E-stability}

The MSV predictor is always efficient if the $\mathrm{AR}(1)$ predictor is relatively more expensive and the $\operatorname{AR}(1)$ solution is never stable under learning $(\beta<1)$. A version of the proposition found in Guse (2003b) shows that for some parameter values, the MSV REE can always be evolutionary E-stable:

Proposition 2: For the above model, the MSV REE is evolutionary E-stable and the AR(1) REE is not evolutionary E-stable for all $\mu \in(0,1]$, when $K_{1}-K_{2}<0$, and $\beta_{0}<1$.

The proof is given in Appendix A. When the MSV REE is evolutionary E-stable for all $\mu \in(0,1]$, the model is said to be MSV dominant. Here, as long as $\mu_{0}>0$, the replicator 
dynamics will direct the entire population to use the MSV predictor. Furthermore, the resulting learned equilibrium will be the MSV REE.

Guse (2003b) does not consider the case where the cost of using the MSV predictor is greater than the cost of using the $\mathrm{AR}(1)$ predictor. This is presented in the following proposition:

Proposition 3: For the above model, the AR(1) REE is evolutionary E-stable and the MSV REE is not evolutionary E-stable for all $\mu \in[0,1)$, when $K_{1}-K_{2}>0, \beta_{0}>1$, and $\beta_{0}+\beta_{1}<1$.

The proof is given in Appendix B. Since the MSE for the AR(1) predictor is always less or equal to the MSE of the MSV predictor, a larger relative cost for using the MSV predictor will make the $\mathrm{AR}(1)$ predictor the efficient predictor for all levels of heterogeneity. When the $\operatorname{AR}(1)$ solution is evolutionary E-stable for all $\mu_{0} \in[0,1)$, I will refer to the model as being AR(1) dominant. This result is not found in Guse (2003b) as a non-zero cost for using the MSV predictor was not considered.

With the possibility of MSV and AR(1) dominance, the above model can be presented in such a manner where the efficient predictor may randomly change due to some structural shock to the economy. For instance, the model may switch from being MSV dominant to AR(1) dominant. I will compare the results of this mechanism using: rational expectations (RE), evolutionary game theory with no econometric learning, and evolutionary game theory with econometric learning.

\subsection{The Markov Process}

Assume that a Markov process occurs within the above model. This process will cause a change of predictor efficiency due to a structural shift to the economy. For example, we may see a change in monetary policy, fiscal policy, technology, or a change in consumer or firm behavior. The most efficient predictor may change from such a shock to the economy.

The time invariant Markov chain presented in this paper will follow Ljungqvist and Sargent (2000). It is defined by a triple of objects:

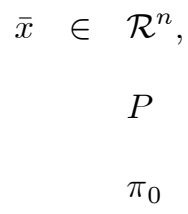


where $\bar{x}$ records the possible values of the states of the system, $\mathrm{P}$ is the transition matrix, and $\pi_{0}$ records the probabilities of being in each state i at time 0 . I make the two standard assumptions below that are also made by Ljungqvist and Sargent (2000).

Assumption 1: For $\mathrm{i}=1, \ldots, \mathrm{n}$, the matrix $\mathrm{P}$ satisfies

$$
\sum_{j=1}^{n} P_{i j}=1
$$

Assumption 2: The vector $\pi_{0}$ satisfies

$$
\sum_{i=1}^{n} \pi_{0 i}=1
$$

Matrix $\mathrm{P}$ is a stochastic matrix which defines the probabilities from moving from one state to any other in one period. It has the interpretation

$$
P_{i j}=\operatorname{Prob}\left(x_{t+1}=\bar{x}_{j} \mid x_{t}=\bar{x}_{i}\right) .
$$

The vector $\pi_{0}$ has the interpretation

$$
\pi_{0 i}=\operatorname{Prob}\left(x_{0}=\bar{x}_{i}\right) .
$$

In the case of the Taylor model with only two predictors, the Markov chain is the following:

$$
\begin{aligned}
x & =\left[\left(\beta_{0}^{A}, \beta_{1}^{A}, K_{1}^{A}, K_{2}^{A}\right),\left(\beta_{0}^{B}, \beta_{1}^{B}, K_{1}^{B}, K_{2}^{B}\right)\right] \\
P & =\left[\begin{array}{ll}
P_{11} & P_{12} \\
P_{21} & P_{22}
\end{array}\right] \\
\pi_{0} & =\left[\pi_{0,1},\left(1-\pi_{0,1}\right)\right]
\end{aligned}
$$


where

$$
\begin{aligned}
K_{1}^{A} & =K_{2}^{B}=0 \\
K_{2}^{A} & =K_{1}^{B}=k \\
k & >0 .
\end{aligned}
$$

To follow the Taylor model, assume that

$$
\begin{aligned}
& \beta_{0}^{A}=-\beta_{1}^{A} \\
& \beta_{0}^{B}=-\beta_{1}^{B} .
\end{aligned}
$$

\subsubsection{State A: The MSV Evolutionary E-stable State}

In state A, the MSV REE is evolutionary E-stable since the MSV updating rule is the efficient predictor. Here there is some cost, $K_{2}^{A}$ for using the AR(1) updating rule such that all of the agents will asymptotically choose the MSV predictor for any $\mu_{0} \in(0,1]$. For this to occur, it must be that $\beta_{0}^{A}<\left(1-\mu_{0}\right)^{-1}$. A natural choice is $\beta_{0}^{A}<1$ and $\beta_{1}^{A}=-\beta_{0}^{A}$ where the MSV REE is E-stable for any level of heterogeneity.

\subsubsection{State B: The AR(1) Evolutionary E-stable State}

State $\mathrm{B}$ is the $\mathrm{AR}(1)$ evolutionary E-stable state where the $\mathrm{AR}(1)$ updating rule is the efficient predictor for all $\mu \in[0,1)$. With the new relative costs, the AR(1) REE will always be evolutionary E-stable if $\mu \neq 1$ and the $\operatorname{AR}(1)$ REE is E-stable. A natural choice for the parameters here are $\beta_{0}^{B}>1$ and $\beta_{1}^{B}=-\beta_{0}^{B}$ where either the MSV or AR(1) MEE is E-stable for all $\mu \in[0,1]$, and the $\mathrm{AR}(1) \mathrm{REE}$ is E-stable when $\mu=0$.

\subsubsection{Intuition for State Changes and Costs}

Although the states represent one of the two predictors being the efficient predictor, a more interesting interpretation is to assume each state brings a new efficient predictor into the economy. Assume that agents receive their predictions from one of two existing forecast agencies. One agency provides predictions from an $\mathrm{AR}(1)$ process with learned parameter values while the other 
provides the predictions from the MSV process with learned parameter values. In every state, one of the two predictors is known as the "new" predictor. In order for the agency with the new predictor to attract new customers, they will charge a lower cost than the other agency. At a state change, the old agency will go out of business leaving room for another to enter the market. Those using the exiting agency's predictor will then be forced to use the only existing agency's predictor. Then, a new agency will enter the market with the exiting agency's predictor with some new initial priors. A very small proportion of agents will see this agency enter the market and will use this new predictor. ${ }^{8}$

\section{Necessary Conditions For Evolution and E-stability}

In order for the above system to be stable, the resulting parameter equilibrium must be stable under learning and the replicator dynamics must asymptotically direct the population to use the efficient predictor. To achieve this, the parameter estimates, $a_{1 t}, a_{2 t}$, and $b_{2 t}$, and the population, $\mu_{t}$, must always be contained within the domain of attraction of the current evolutionary E-stable REE, $\phi\left(\mu^{*}\right)$. Consider the following restrictions that must be made in order to ensure stability within the two states.

Assume that the model switches from state B to state $\mathrm{A}$ at time $t=T$. If no agents are using the new MSV predictor at this time, then the level of heterogeneity will never change by the replicator dynamics. When the model moves to state A, let

$$
\mu_{T}=\mu_{L}>0
$$

where $\mu_{L}$ denotes the proportion of intelligent agents who instantaneously discover that the MSV predictor is efficient at the time of the state switch. This allows some agents a larger degree of intelligence than others. Assume that there is a subset of agents who do not posses RE, but they are able to determine the (asymptotic) efficiency of each updating rule at any time, $t$. All agents may be able to witness a structural change in the economy, but only these agents see that

\footnotetext{
${ }^{8}$ Although the new agency always provides the most efficient predictor, this is only asymptotically. In the short run, as this agency learns, the MSE for this predictor may be higher than that of the old agency's prediction. Therefore, it would not be beneficial for all agents to use the new predictor at the initial state change.
} 
this change affects the efficiency of each predictor. Therefore, these agents rationally change their predictor to the efficient predictor at time $T$.

Now suppose the model switches from state $\mathrm{A}$ to state $\mathrm{B}$ at time $t=T$. Once again, if no agents use the new $\operatorname{AR}(1)$ predictor, then the level of heterogeneity will never change by the replicator dynamics. Therefore, the more intelligent agents choose the AR(1) updating rule at the state change such that

$$
\mu_{T}=\mu_{H}<1
$$

Note that at the time of the state change, the $\operatorname{AR}(1)$ predictor may receive new priors. Since the $\mathrm{AR}(1)$ solution is not globally stable under learning, it must be that the new priors are inside the basin of attraction of the E-stable equilibrium. As discussed in chapter 6 of Evans and Honkapohja (2001), a reasonable set of priors, within the basin of attraction, and shocks with a relatively small support should be enough to ensure stability. ${ }^{9}$

\section{Learning Within and Between States}

As in previous literature, agents will learn the parameter values of the model using recursive least squares within each state. Evans and Honkapohja (2001) show that this system of learning can be written as a stochastic recursive algorithm (SRA):

$$
\theta_{t}=\theta_{t-1}+\gamma_{t} \mathcal{H}\left(\theta_{t-1}, X_{t}\right)
$$

where $\theta_{t}$ is a vector of parameter estimates: ${ }^{10}$

$$
\theta_{t}=\operatorname{vec}\left(\phi_{t} S_{t} M S E_{t}\right)
$$

$\phi_{t}=\left(a_{1 t}, a_{2 t}, b_{2 t}\right)^{\prime}, S_{t}$ is the moment matrix for AR(1) estimation, $M S E_{t}=\left(M S E_{1 t}, M S E_{2 t}\right)^{\prime}$, $X_{t}$ is the state vector, $\mathcal{H}($.$) is a function describing how the vector \theta$ is updated, and $\gamma_{t}$ is a deterministic positive, nonstochastic, nonincreasing sequence of "gains." Previous literature has

\footnotetext{
${ }^{9}$ A projection facility may alternatively be used, however, it has been criticized in Grandmont and Laroque (1991), Grandmont (1998), and Moreno and Walker (1994) as being inappropriate for decentralized markets.

${ }^{10} v e c$ is a matrix operator which stacks, in order, the columns of the matrix $\left(\phi_{t} S_{t} M S E_{t}\right)$ into a column vector.
} 
assumed that

$$
\sum_{t=1}^{\infty} \gamma_{t}=\infty \text { and } \sum_{t=1}^{\infty} \gamma_{t}^{2}<\infty
$$

As time goes by, the present becomes less important in the updating of parameter values. This causes parameter values to converge asymptotically as long as the initial conditions are contained within the basin of attraction of the equilibrium.

Learning within a state will be very similar to previous literature. Agents will continue to update the parameter estimates using recursive least squares. This system can be written using the above SRA. Within a state, E-stability conditions will hold since the gain parameter will diminish over time.

At a state change agents using the new efficient updating rule should not emphasize the past as they did with the other updating rule. ${ }^{11}$ The gain parameter will not converge to zero when the model switches between equilibria between states. In this way, once agents learn of the switch, they will no longer put such a large emphasis on the past data since they are aware of the recent structural change to the economy. ${ }^{12}$

\subsection{Updating the Gain Parameter Between States}

Updating the gain parameter at each state change will be similar to the restarting gain technique used by Timmermann (1996). When a state switch occurs, at $t=T$, agents who use the new efficient predictor, $i$, will adjust the gain parameter to

$$
\gamma_{i T}=\frac{1}{\omega}
$$

The gain sequence will follow like recursive least squares

$$
\gamma_{i, t+1}=\frac{\gamma_{i, t}}{1+\gamma_{i, t}}
$$

\footnotetext{
${ }^{11}$ Agents are not aware of the state change until they switch to the efficient predictor. Since they do not know when the economy is in each state, they can not use information from past states to form expectations.

12 This process, with a decreasing gain parameter, produces results similar to "escape dynamics" discussed in Sargent (1999), Williams (2003b) and Cho, Williams, and Sargent (2002) who assume a constant gain parameter.
} 
however, at any time before another state change, if

$$
M S E_{i t}<(1+\varkappa) * \sigma^{2}
$$

where $\varkappa>0$, then the gain sequence will be restarted to

$$
\gamma_{i, t+1}=\frac{1}{\omega}
$$

With the introduction of the confidence variable in aggregate demand, even a small increase in the average $M S E$ (decrease in confidence) can lead to a large change in output and prices. ${ }^{13}$ Those using the new predictor are aware of this and therefore, they aggressively learn using a constant gain learning algorithm. Aggressive learning will continue until the agents are convinced that they have learned the equilibrium. Agents using the old predictor are not aware of the state change and therefore, do not restart their gain sequence and continue to use an infinite memory learning algorithm.

At any time $t$, there exists a gain vector,

$$
\gamma_{t}=\left(\gamma_{1 t}, \gamma_{2 t}\right)
$$

where

$$
\gamma_{1 t}<\gamma_{2 t}
$$

in state $\mathrm{A}$ and

$$
\gamma_{2 t}<\gamma_{1 t}
$$

in state B. ${ }^{14,15}$ The new SRA becomes

$$
\theta_{t}=\theta_{t-1}+\gamma_{t} \mathcal{H}\left(\theta_{t-1}, X_{t}\right) .
$$

\footnotetext{
${ }^{13}$ Too large of a $\kappa_{3}$ can destabilize the system in which an increase in the average MSE can lead to larger and larger values for the intercept term, $\alpha_{t}$ from equation (1). The system seems to be stable given my choice of $\kappa_{3}$.

${ }^{14}$ Using the E-stability results from Giannitsarou (2003) and Guse (2004a), it can be inferred that the E-stable equilibrium will be stable under learning even when agents have different gains and different PLM's.

${ }^{15}$ Here, the gain parameters are random. Random gains that do not converge to zero have been discussed in Evans, Honkapohja, and Marimon (2001) and Honkapohja and Mitra (2003).
} 
Within a state (assuming that there is no switch to the other state), each gain sequence has the same asymptotic property as the previous literature where

$$
\lim _{t \rightarrow \infty} \gamma_{i t}=0
$$

Therefore, learning has the same asymptotic "feel" in each state, but this property will not appear between states. The probability of staying in the same state forever in the Markov chain is zero, therefore, within the entire model:

$$
\lim _{t \rightarrow \infty} \gamma_{i t} \neq 0
$$

since

$$
\operatorname{prob}\left(\gamma_{i t}=\omega^{-1} \mid S(t-1)=j\right)=p_{j i}>0
$$

and

$$
\operatorname{prob}\left(\gamma_{i, t+1}=\omega^{-1} \mid S(t-1)=i\right)=p_{i j} p_{j i}>0
$$

Now, within the model, there will not be convergence to a single equilibrium, but there will be a tendency of convergence to a single equilibrium within each state.

\section{Dynamics}

Next, I analyze the dynamics of the model under three conditions. First, the dynamics will be discussed under rational expectations. This is the case where at the time of the state change, all of the agents instantaneously change to the new efficient predictor. Next, I will discuss the dynamics of the case of "fast-slow" learning. This is the case where the variables of the MEE are learned infinitely fast compared to the replicator dynamics. Finally, I consider the case of econometric learning with replicator dynamics. I show that a less rational system accentuates asymmetry in an Expectational Business Cycle. 


\subsection{Confidence}

In order to show a business cycle, from a change in uncertainty, output must be negatively dependent upon uncertainty. Assume that agents receive utility based upon how well they form expectations. Agents who are more certain about the status of the current economy will consume and invest more than those less certain of the status of the economy. More informed agents will be able to distinguish between permanent and transitory shocks and thus will be able to make better decisions than less informed agents. The Mean Squared Error will be used as a proxy for ability to form expectations.

The average level of confidence in the economy will be the average of the inverses of MSE's realized in the previous period:

$$
C_{t}=\mu_{t} \frac{1}{M S E_{1, t-1}}+\left(1-\mu_{t}\right) \frac{1}{M S E_{2, t-1}}
$$

As agents learn the REE, confidence will tend to increase, so at a state change, the level of confidence will tend to decrease and then increase as agents learn the REE.

\subsection{Rational Expectations}

Consider the case of Rational Expectations where all agents know that the state switch occurred and instantaneously start using the corresponding efficient predictor at time $t=T$. In this case, confidence will be

$$
C_{t}=\frac{1}{\sigma^{2}}
$$

in either state at all times. In this case, real GDP, besides white noise, will remain constant throughout time. This demonstrates that the Markov process is not creating any fluctuations in output, so if business cycle-like dynamics are to occur, then it must be from agents changing the way they form expectations. I will show this in both the case of replicator dynamics without learning and the case of replicator dynamics with learning. 


\subsection{Replicator Dynamics without Learning}

Next, consider the case where agents learn parameter values infinitely fast compared to the replicator dynamics. At every period, the agents know the MEE, but will not necessarily know which of the two predictors is efficient. Predictor choice will be dictated by the replicator dynamics directing agents to use the efficient predictor Because agents know the MEE, GDP is determined by confidence and the stochastic shocks.

First, consider the dynamics in state A where the MSV REE is evolutionary E-stable. Each predictor produces the following MSE: ${ }^{16}$

$$
M S E_{1}=M S E_{2}=\sigma^{2} .
$$

Therefore, the level of confidence in the economy is:

$$
C_{t}=\mu_{t} \frac{1}{\sigma^{2}}+\left(1-\mu_{t}\right) \frac{1}{\sigma^{2}}=\frac{1}{\sigma^{2}} .
$$

With no learning, the level of confidence does not change through time, so real GDP will only change from white noise and no business cycle-like dynamics are produced.

Next, consider the dynamics in state B where the AR(1) REE is evolutionary E-stable. When the MSV MEE is E-stable, confidence is the same as it is in state 1 because the MSE's are the same for each predictor. However, when the level of $\mu$ moves to the point where

$$
\mu<1-\frac{1}{\beta_{0}},
$$

then the AR(1) MEE becomes the E-stable solution and $M S E_{1}>M S E_{2}$. The level of confidence in the economy for $\mu<1-\frac{1}{\beta_{0}}$ in state $\mathrm{B}$ is the following:

$$
C_{t}=\left(1-\mu_{t}\right) \frac{1}{\sigma^{2}}+\mu_{t}\left(\frac{1}{\sigma^{2}}\left(1-\frac{\left(1-\left(1-\mu_{t}\right) \beta_{0}\right)^{2}}{\left(1-\mu_{t}\right)^{2} \beta_{1}^{2}}\right)\right)<\frac{1}{\sigma^{2}} .
$$

\footnotetext{
${ }^{16}$ Theoretical $M S E^{\prime} s$ are presented in appendix C.
} 
For $\mu \in\left[0,1-\frac{1}{\beta_{0}}\right)$, the level of confidence has a local minimum at

$$
\mu_{M}=\frac{1+2 \beta_{0}-\sqrt{1+8 \beta_{0}}}{2 \beta_{0}}
$$

When $\mu$ is still relatively high, confidence will decrease as $\mu$ falls since $M S E_{1}$ is increasing. However, average confidence will begin to increase as less agents use the MSV predictor. Therefore, as $\mu$ decreases with the replicator dynamics, confidence first stays constant at $C_{t}=\frac{1}{\sigma^{2}}$, then decreases, and then returns to:

$$
C_{t}=\frac{1}{\sigma^{2}}
$$

The results for $y$ are expressed on figure 1. Since agents know the MEE for all time periods, $y$ is entirely determined by the level of confidence (and an error term in which I leave out for presentation). At time zero, there is a state change from A to B. The level of heterogeneity, $\mu$, decreases when the intelligent agents switch to using the AR(1) updating rule. GDP does not initially decrease as confidence does not initially decrease. However, when $\mu<1-\frac{1}{\beta_{0}}$, output begins to decline as confidence declines. When $\mu<\mu_{M}$, output increases with confidence and asymptotically returns to the REE level $(y=0)$. By simple inspection, one can see that the expectational business cycle is only slightly asymmetric and the decline in output is rather small. When agents learn the parameters, it turns out that this asymmetry becomes much more obvious and the decline in output becomes much larger.

FIGURE 1. Expectational Business Cycle -AR(1)

Replicator Dynamics with no Learning 

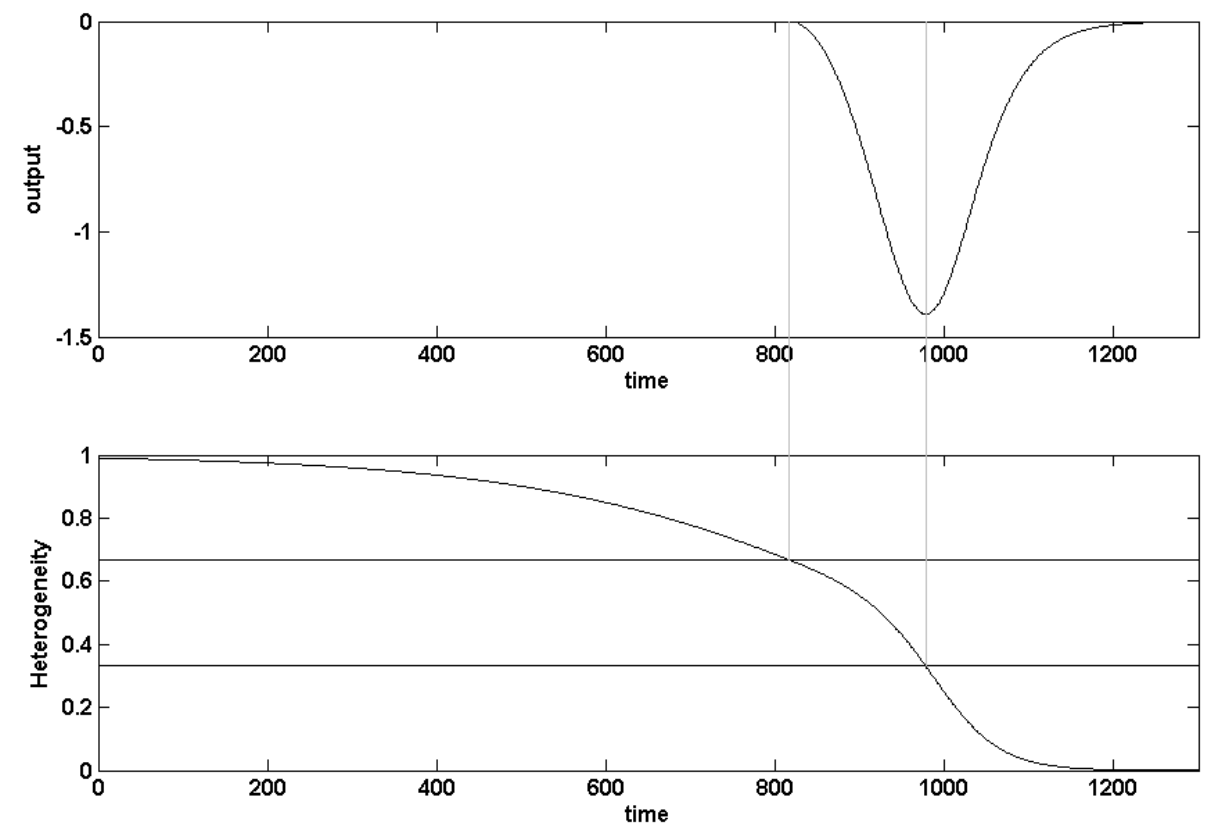

With no learning, the state change from A to B first has no affect on confidence, but confidence changes as the AR(1) MEE becomes the E-stable equilibrium. A change in the efficient predictor led to an "expectational business cycle" within the state from the replicator dynamics and a change in the learnable equilibrium. This type of "business cycle" exists because of the replicator dynamics and not the Markov process.

\subsection{Replicator Dynamics with Learning}

In the previous section, a change to state A did not produce an "expectational business cycle." This was due to agents having rational expectations. A more interesting result would be the possibility of a decrease in confidence from such a state change. Econometric learning creates such properties for this model. This section will show that the existence of learning may create a further possibility of an "expectational business cycle."

Consider the following learning algorithm:

$$
\theta_{t}=\theta_{t-1}+\gamma_{t} \mathcal{H}\left(\theta_{t-1}, X_{t}\right)
$$


where $\theta_{t}=\operatorname{vec}\left(\phi_{t} S_{t} M S E_{t}\right)$ and $M S E_{t}^{\prime}=\left(M S E_{1 t}, M S E_{2 t}\right)$. The first two components of this SRA are

$$
\begin{aligned}
& \phi_{t}=\phi_{t-1}+\gamma_{t}\left(S_{t-1}^{-1} z_{t-1}\left(y_{t}-z_{t-1}^{\prime}\left(\begin{array}{c}
a_{2, t-1}-a_{1, t-1}-\rho v_{t-1} \\
b_{2, t-1}
\end{array}\right)-\rho v_{t-1}\right)\right) \\
& S_{t}=S_{t-1}+\gamma_{t}\left(z_{t} z_{t}^{\prime}-S_{t-1}\right)
\end{aligned}
$$

where

$$
\begin{aligned}
\phi_{t-1}^{\prime} & =\left(a_{1, t-1}, a_{2, t-1}, b_{t-1}\right) \\
z_{t-1}^{\prime} & =\left(1, y_{t-1}\right) .
\end{aligned}
$$

The MSE component of the SRA is the following:

$$
\left.\begin{array}{rl}
M S E_{t}= & M S E_{t-1}+ \\
& \gamma_{m} *\left(\left(\left(p_{t}-z_{t-1}^{\prime}\left(\begin{array}{c}
\left(p_{t, t-1}-a_{1, t-1}\right)^{2} \\
b_{2, t-1}
\end{array}\right)\right)^{2}\right)-M S E_{t-1}\right.
\end{array}\right)
$$

where $\gamma_{m}>0$ is a fixed gain parameter. $M S E_{t}$ demonstrates how well the learned parameters, for each predictor, have predicted $p_{t}$. It is important since the confidence variable, $C_{t}$, is based upon the ability to predict. The agents do not learn this value, but they do receive the corresponding level of uncertainty from it. Since this value is not learned by the agents, it will be generated by a finite memory algorithm. Equation (11) will provide different dynamics of confidence and output in state A than what was shown with no learning.

\subsubsection{State B to State A}

FIGURE 2. Expectational Business Cycle-MSV

Replicator Dynamics with Learning 

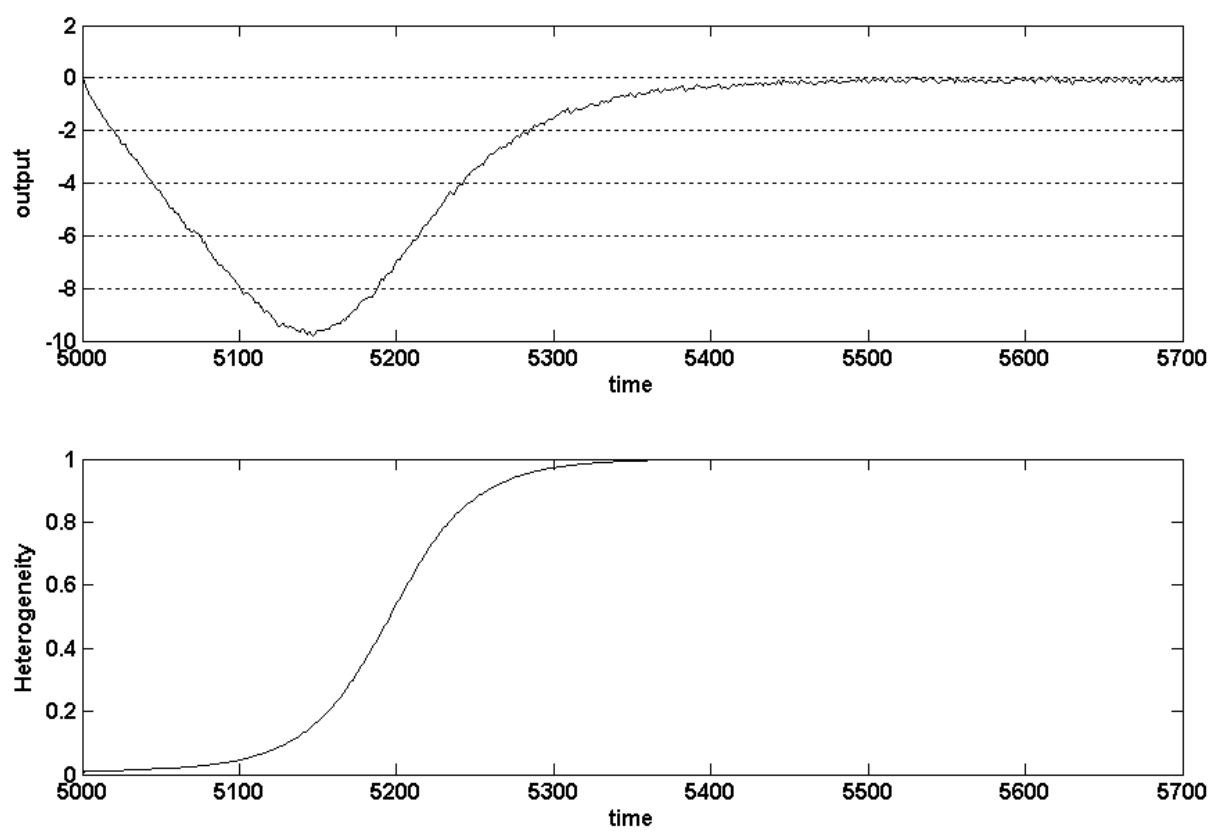

First, consider the dynamics from a change from state B to state A. Recall that at this change, the MSV updating rule becomes the efficient predictor over the $\mathrm{AR}(1)$ updating rule. For simplicity, assume that at $t=T_{1}-1$, the agents have learned the $\operatorname{AR}(1) \operatorname{REE}$, and $\mu=0$. This means that $M S E_{2}=\sigma^{2}$, so the level of confidence is equal to:

$$
C_{t}=\frac{1}{\sigma^{2}} \text {. }
$$

At time $t=T_{1}$, assume that a small amount of agents, $\mu=\mu_{L}$, see the state change and decide to use the MSV predictor and

$$
M S E_{T_{1}}=\left(\begin{array}{c}
\sigma^{2} \\
\sigma^{2}
\end{array}\right)
$$

as it is in the case with no learning.

Learning will now change the dynamics of the level of confidence through time. Consider the replicator dynamics at $t=T_{1}$ :

$$
\mu_{T_{1}+1}=\mu_{L}\left(\frac{\frac{1}{\sigma^{2}}}{\mu_{L}\left(\frac{1}{\sigma^{2}}\right)+\left(1-\mu_{L}\right) *\left(\frac{1}{\sigma^{2}}-K_{2}^{A}\right)}\right) .
$$


Since $K_{2}^{A}>0$, there will be more agents using the MSV predictor at $t=T_{1}+1^{17}$. The agents will continue to choose the MSV predictor over the $\mathrm{AR}(1)$ predictor until all agents are using the MSV predictor. Those using the MSV predictor are updating their estimates with a larger gain. Therefore, $a_{2}$ and $b_{2}$ parameters will be updated relatively slowly and thus the updated $M S E_{2}$ will increase. As long as the learned parameters for $a_{2}$ and $b_{2}$ are not equal to $a_{1}$ and 0 respectively, the $M S E_{2}$ will continue to increase. An increase in the $M S E_{2}$ will decrease $C_{t}$ and thus increase $\alpha_{t}$ in equation (1). This then increases $M S E_{1}$ and further increases $\alpha_{t}$. With aggressive learning, the agents using the efficient updating rule prevent $\alpha_{t}$ from diverging to infinity. As more agents use the efficient predictor, and the agents are more certain of the updating rule, the value of $\alpha_{t}$ decreases and converges to $\alpha_{t}=m$, the REE value.

Figure 2 presents the dynamics of output and $\mu$ when the MSV updating rule becomes the efficient predictor. It is a result of average of 1000 simulation results where the economy is in state B for the first 5000 periods and is in state A for the next 5000 periods. The values used for all the simulations are: $\kappa_{2}=2, \kappa_{3}=20, \phi_{1}=3.2, \lambda_{2}=.6, \zeta=20$, and $\sigma^{2}=1$. Since $\beta_{0}$ is homogeneous of degree 1 with respect to $\lambda_{1}$ and $\kappa_{1}$ and the coefficients on the error terms are homogeneous of degree zero with respect to the same variables, I change only these values between the two states In state A, $\kappa_{1}=\lambda_{1}=2$ so that $\beta_{0}^{A}=0.5$, and in state B, $\kappa_{1}=\lambda_{1}=12$, so that $\beta_{0}^{B}=3$.

In figure 2, output decreases for the first 150 periods with a small increase in agents using the efficient predictor. This large change in output comes from an increase in both $M S E^{\prime} s$ as agents are learning the new equilibrium. The replicator dynamics direct all the agents towards using the MSV updating rule. As more agents use this rule, $C_{t}$ increases and thus output increases as well. However, since agents are still learning, the expansion back to equilibrium is slower than the initial recession. Therefore, the combination of learning and replicator dynamics produce an asymmetric Expectational Business Cycle.

\footnotetext{
${ }^{17}$ Since I am looking at real-time dynamics, there is a possibility that $U_{1}<U_{2}$ from the estimated MSE's. In this case, assume that the agents using the MSV predictor know their future utility will be greater than the utility of using the AR(1) predictor, thus they will choose to keep using the asymptotically "efficient" predictor.
} 


\subsubsection{State A to State B}

FIGURE 3. Expectational Business Cycle-AR(1)

Replicator Dynamics with Learning

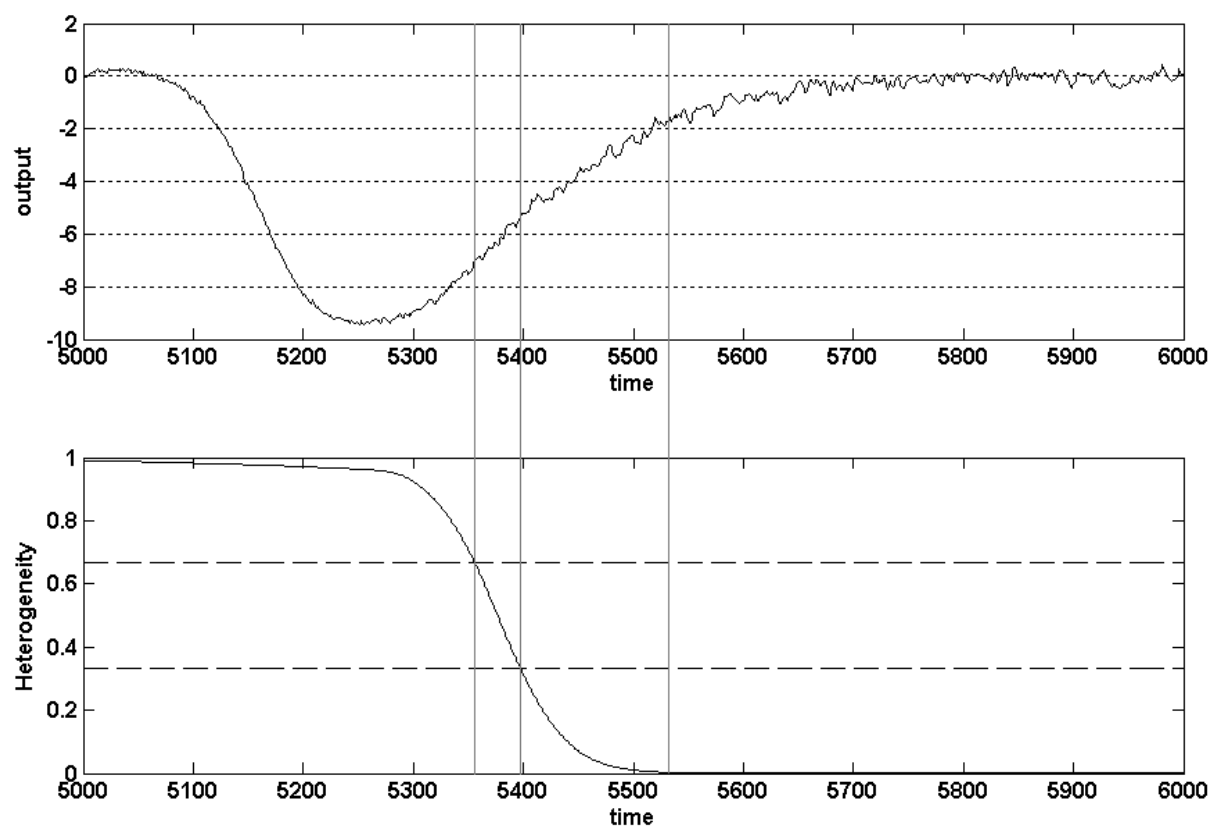

Next, I examine the dynamics from a change to state B where the $\mathrm{AR}(1)$ updating rule becomes the efficient predictor. Assume that the economy has reached the equilibrium levels prior to this state change so that $\mu=1$ and $C_{t}=\frac{1}{\sigma^{2}}$. At time $T_{2}$, assume that a small proportion of agents, $\left(1-\mu_{H}\right)$ see the state change and switch to using the $\operatorname{AR}(1)$ updating rule. Here,

$$
M S E_{T_{2}}=\left(\begin{array}{c}
\sigma^{2} \\
\sigma^{2}
\end{array}\right)
$$

as it is in the case with no learning.

Next consider the replicator dynamics when there is a cost for using the MSV predictor of 
$K_{1}^{B}>0$ at $t=T_{2}$ :

$$
\mu_{T_{2}+1}=\left(1-\mu_{L}\right)\left(\frac{\frac{1}{\sigma^{2}}}{\left(1-\mu_{L}\right)\left(\frac{1}{\sigma^{2}}-K_{1}^{B}\right)+\mu_{L} *\left(\frac{1}{\sigma^{2}}\right)}\right) .
$$

The proportion of agents using the $\mathrm{AR}(1)$ predictor will increase as times goes by. Those now using the $\mathrm{AR}(1)$ updating rule are learning more aggressively than the agents using the MSV updating rule. Small shocks to the system can lead to those using the AR(1) updating rule to believe that $b_{2} \neq 0$ even though the MSV MEE is E-stable for $\mu>1-\frac{1}{\beta_{0}}$. As a result, $M S E_{2}$ may increase leading to an increase in $\alpha_{t}$. This will then in turn increase $M S E_{1}$ as these agents are not learning as aggressively as the AR(1) learners. The process of learning thus leads to an overall decrease in the level of confidence, $C_{t}$ and a decrease in output. As less agents use the MSV learning rule, the level of confidence and output will increase back to their equilibrium levels.

Figure 3 shows that the real-time learning dynamics of the model in state B are much different than the dynamics without learning. After the state change, there is a rather short period small increase in output. This occurs due to the empirical MSE's being smaller than $\sigma^{2}$. However, as this goes away, output reduces rather quickly when the majority of agents are still using the MSV updating rule. Using learning and an estimated MSE results in a faster arrival of a recession than with RE and replicator dynamics. The expected arrival time of the recession is shown by the first vertical gray line in figure 3. Learning also extends the length of the recession. In figure 3 , the expected length of the recession is the distance between the first and second gray lines. However, one can see that the length between the first peak and the valley is much greater. By comparing figure 3 with figure 1 , one can see how learning greatly accentuates the asymmetry in the Expectational Business Cycle. In figure 1, the recovery is just a little longer than the decline, but with learning, figure 3 shows that the recovery is approximately 3 times longer than the decline.

\subsubsection{The Markov Process}

Next, figure 4 shows the dynamics of the system under a Markov Process. The parameter values in states A and B are the same as above and the model arbitrarily initially starts in state A. 
Within each state, the probability of entering the other state is .03\% meaning that 3 state changes should occur on average for a simulation of 10,000 periods. A simulation of the Markov process was first run for 10,000 periods. Then, using this process, 1000 simulations each consisting of 10,000 periods was run to smooth the data as was done above.

Figure 4 shows that the Markov process can affect the length of both the expansions and the recessions. There is a long period of expansion after the recession caused by the first state change due to a long period of staying in state B. When there is no change in the efficient predictor, learning allows agents to unknowingly coordinate to the REE. When the agents learn the REE, they become quite confident about their ability to forecast and thus the random i.i.d. shocks are dictating the dynamics of the economy.

There is a substantially smaller time period between the second and third state changes. After the second state change, agents do not have enough time to learn the new social norm predictor and do not learn the REE before the third state change. As a result, agents are quite confused about which predictor is efficient. A recession may last quite a long time on the unlikely result of several state changes over a short time period.

The recessions as a result of a switch to state B are on average much larger than those from state A. This results from the fact that the price level is not a stationary system in the short run due to the change in MSE's. At the change to state B, the initial proportion of agents who use the MSV updating rule is set at .99. A small shock to the economy can lead to a higher average MSE. The average MSE feeds back into the system making it no longer stationary since the constant is now dependent on the average MSE. This further increases the MSE for using the MSV rule. ${ }^{18}$ The replicator dynamics directs agents using the MSV updating rule to use the $\mathrm{AR}(1)$ rule. As more agents us the $\mathrm{AR}(1)$ rule, the effect from the higher MSE diminishes and the process becomes stationary again. This process does not typically occur during the transition dynamics in state A because most of the agents initially use an updating rule that can learn a non-stationary equilibrium. During these dynamics, when most of the agents are using the MSV rule, the recession has ended and thus the system is again stationary.

\footnotetext{
${ }^{18} \mathrm{~A}$ large value of $\kappa_{3}$ can lead to the process becoming highly unstationary in the short run and thus a non-stable system. Assuming a small enough $\kappa_{3}$ is equivalent to assuming a stationary solution.
} 
FIGURE 4. The Full System
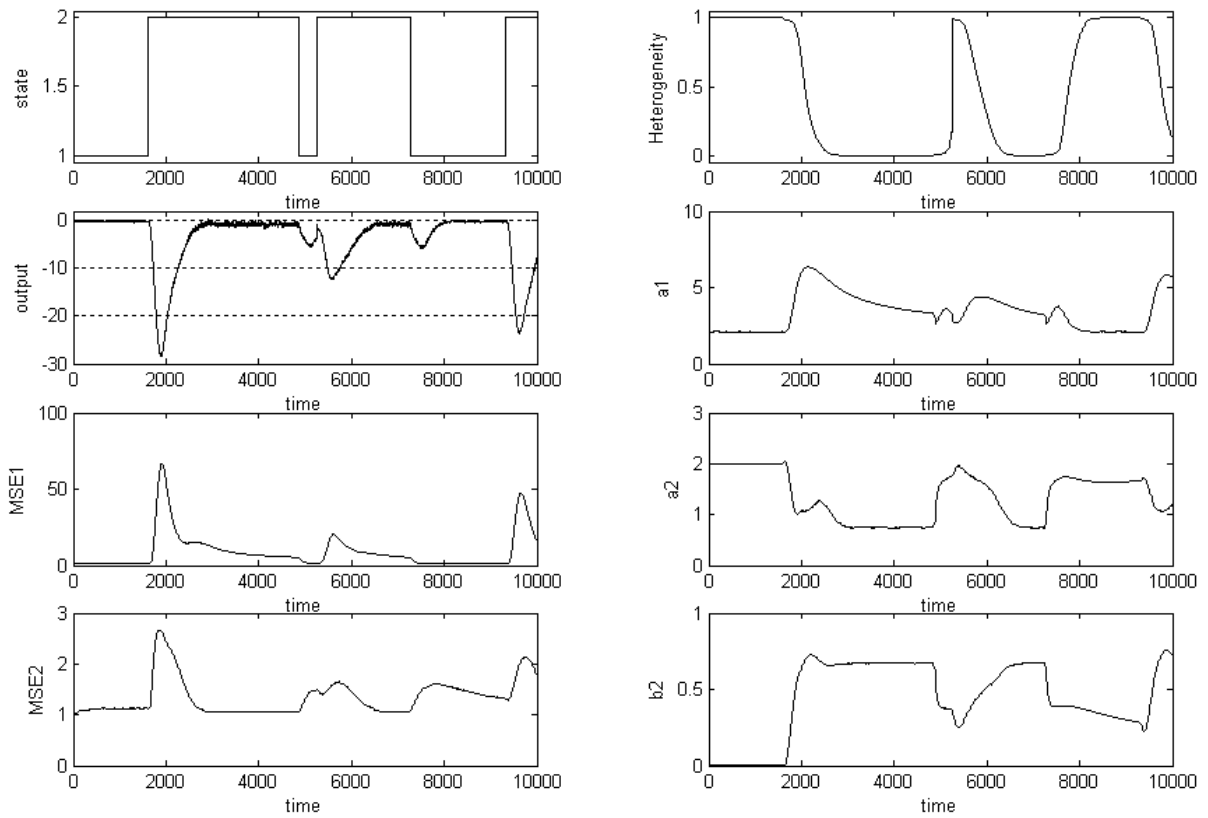

\subsubsection{Discussion of Results}

Hamilton (1989) presents a model where the parameters of an autoregression are an outcome a Markov process. Here an econometrician must make an inference of the unobserved state based on the observed behavior of the economy. Hamilton presents an empirical application to his technique where there is a two state process: one state is positive growth while the other state is negative growth which is a recurrent feature of the U.S. business cycle. He finds that a typical recession is associated with a $3 \%$ permanent drop in the level of GNP.

The above model is similar as it incorporates a Markov process, however, it is not intended for estimation. For explaining the dynamics of a business cycle, this technique is more realistic than Hamilton's for two reasons. First, recessions and expansions are dictated by expectations and learning dynamics and not some exogenous state change. Recall the relationship between business cycles and expectations which suggests that learning may be quite an important mechanism when describing the business cycle. Second, Hamilton's technique suggests that a long 
recession or very short recession may be more common than suggested by the above model. This is due to the process being driven by exogenous state changes and not an endogenous propagation mechanism. Above, a recession occurs as a response to a state change, but dissipates as agents learn the new equilibrium. Under "normal" circumstances, a very short recession could not occur while a long recession could only occur due to several unlikely state changes.

I have shown that as rationality decreases, the effect from a state change to aggregate output is greatly magnified. First, the arrival time of the decline in output is shortened when agents learn the parameters of the model and the cycle takes longer to return to the equilibrium value. Second, the overall decline itself is much greater than it is without learning and only the replicator dynamics. These results show how limited information can negatively affect the economy. Uninformed, risk-averse agents will tend to produce and consume less than what they would with more information. In the model above, suppose that there was an outside agent that provided additional (correct) information. With this additional information, agents can learn and discover the best way to learn faster than without the information. By providing the additional information, the outside agent could prevent such large downswings of aggregate output due to uncertainty.

\section{Conclusion}

This paper has developed a model of business cycles through the process of learning and replicator dynamics. The model used is a simple self referential linear stochastic model discussed in Taylor (1977). This model is provided as a starting point to introduce the concept of Expectational Business Cycles. I assumed a stationary process for the model for any $\mu$, the proportion of agents who use the MSV predictor, such that stability properties will always occur in the model. From this assumption, it follows that the results in Guse (2003b) can be used to determine stability under learning and evolutionary dynamics of the equilibria in the model. The efficient predictor in the model changes randomly via a two-state Markov process.

The stability properties of the model are dependent upon the initial value of $\mu$, so the initial values of certain parameters are restricted after a state change. These changes arise from an assumption that agents change their guess of the "efficient" predictor when they see a state 
change, and some "intelligent" agents can see every state change instantaneously. Next, I allow the gain parameter used for learning to adjust in order to have stable learning dynamics within each state.

I discuss the theoretical dynamics of the model within three frameworks. To do this, the confidence level is defined such that it will change through time with changes in uncertainty. The case of rational expectations is first considered which shows that aggregate output does not change between each state change. This means that the Markov process alone does not generate any business cycles. Next, the case of replicator dynamics without learning is considered. Here, the transitional dynamics provide aggregate fluctuations like that of a business cycle in one of the two state changes. When learning is included in the model, these aggregate fluctuations are further accentuated. The arrival time of the expectational business cycle is shortened and the overall decrease in output due to uncertainty is greatly increased. These results suggest the importance of providing information to agents who make decisions based on uncertainty. If the overall uncertainty is minimized, then the decrease of output due to uncertainly should be minimized as well. 


\section{Appendix A. Proof of Proposition 2}

Guse (2003a) shows that the MSV MEE is E-stable for all $\mu \in(0,1]$ when $\beta_{0}<1$ and $\beta_{0}=-\beta_{1}$. Here, the AR(1) MEE is never E-stable. Without loss of generality, assume that $K_{1}=0$ and $K_{2}>0$. When the cost for using the $\mathrm{AR}(1)$ predictor is $K_{2}>0$, the replicator dynamics is (assuming $\zeta=0$ )

$$
\mu_{t}=\frac{\frac{1}{\sigma^{2}}}{\frac{1}{\sigma^{2}}-\left(1-\mu_{t-1}\right) K_{2}} \mu_{t-1} .
$$

For $\mu \in(0,1)$, this is an increasing function, so with the nature of the replicator dynamics, it follows that:

$$
\lim _{t \rightarrow \infty} \mu_{t}=1
$$

Therefore, the MSV REE is evolutionary E-stable and the AR(1) REE is never evolutionary E-stable for all $\mu \in[0,1)$, when $\beta_{0}<1$ and $K_{1}-K_{2}<0$.

\section{Appendix B. Proof of Proposition 3}

Guse (2003a) shows that for $\beta_{0}+\beta_{1}<1$, the AR(1) mixed expectations equilibrium (MEE) is E-stable for all $\mu \in\left[0,1-\frac{1}{\beta_{0}}\right)$ and the MSV MEE is E-stable for all $\mu \in\left[1-\frac{1}{\beta_{0}}, 1\right]$. If $\mu=1-\frac{1}{\beta_{0}}$, the MSV and AR(1) MEE are equivalent at:

$$
\begin{aligned}
& a_{1}=\alpha \\
& a_{2}=\alpha \\
& b_{2}=0 .
\end{aligned}
$$

Here, there is a natural exchange of E-stability from MSV to AR(1) when $\mu$ decreases from 1 to 0. Guse (2003a) shows that at this point of E-stability exchange,

$$
M S E_{1}=M S E_{2}
$$

Without loss of generality, assume that $K_{2}=0$ and $K_{1}>0$. For $\mu>1-\frac{1}{\beta_{0}}$, assume that 
$\zeta=0$. The replicator dynamics becomes

$$
\mu_{t}=\frac{\frac{1}{\sigma^{2}}-K_{1}}{\frac{1}{\sigma^{2}}-\mu_{t-1} K_{1}} \mu_{t-1}
$$

It can be easily seen that this is a decreasing function for $\mu \in\left(1-\frac{1}{\beta_{0}}, 1\right)$, so $\mu_{t}$ will tend to decrease as $t \rightarrow \infty$.

For $\mu<1-\frac{1}{\beta_{0}}$, it turns out that

$$
M S E_{1}>M S E_{2}
$$

for all $\mu \in\left[0,1-\frac{1}{\beta_{0}}\right)$. Here, the replicator dynamics is also a decreasing function. From the nature of the replicator dynamics, it turns out that

$$
\lim _{t \rightarrow \infty} \mu_{t}=0 .
$$

Therefore, for any $\mu_{0} \in[0,1)$ the $\mathrm{AR}(1) \mathrm{REE}$ is evolutionary E-stable and the MSV REE is never evolutionary E-stable.

\section{Appendix C. Calculation of the MSE for both of the PLM's}

\section{MSE for the first PLM}

PLM1:

$$
\begin{aligned}
M S E_{1} & =E\left(y-a_{1}\right)^{2} \\
& =E(y-E(y))^{2} \\
& =\operatorname{Var}(y) \\
& =\frac{\sigma_{v}^{2}}{1-b^{2}}
\end{aligned}
$$

If $b=0$ then the MSE from the first predictor becomes:

$$
M S E_{1}=\sigma_{v}^{2}
$$


When we enter the MEE values in for the $M S E_{1}$ we get the following solution:

$$
M S E_{1}=\frac{(1-\mu)^{2} \sigma_{v}^{2} \beta_{1}^{2}}{(1-\mu)^{2} \beta_{1}^{2}-\left(1-(1-\mu) \beta_{0}\right)^{2}}
$$

\section{MSE for the second PLM}

PLM2:

$$
\begin{aligned}
M S E_{2} & =E\left(y-a_{2}-b_{2} y_{t-1}\right)^{2} \\
& =E\left(T_{a 2}+T_{b 2} y_{t-1}+v_{t}-a_{2}-b_{2} y_{t-1}\right)^{2} \\
& =\sigma_{v}^{2}
\end{aligned}
$$

The mean square error for the second predictor will always be $\sigma^{2}$ as long as y follows a stationary process. This means that the $M S E_{1} \geq M S E_{2}$ for all E-stable stationary values of $\alpha, \beta_{0}$, and $\beta_{1}$. This intuitively makes sense because the $\mathrm{AR}(1)$ predictor is always unbiased while the MSV predictor is unbiased only when $b_{2}=0$. 


\section{References}

[1] Chalkley, M. \& I.H. Lee (1998) Learning and Asymmetric Business Cycles.Review of Economic Dynamics, 1, 623-645.

[2] Cho, I., N. Williams, \& T.J. Sargent (2002) Escaping Nash Inflation Review of Studies, 69, $1-40$.

[3] Cogley,T. (2002) How Fast Can the New Economy Grow? A Bayesian Analysis of the Evolution of Trend Growth. Mimeo.

[4] Evans, G.W., Honkapohja, S., 2001. Learning and Expectations in Macroeconomics. Princeton University Press, Princeton, NJ.

[5] Evans, G.W., Honkapohja, S., Marimon, R., 2001. Convergence in Monetary Inflation Models with Heterogenous Learning Rules. Macroeconomic Dynamics 5, 1-31.

[6] Evans, G.W., S. Honkapohja, \& P. Romer (1998) Growth Cycles. American Economic Review, $88,495-515$.

[7] Giannitsarou, C., 2003. Heterogeneous Learning. Review of Economic Dynamics, 6, 885-906.

[8] González, F.M. (1997) Individual Experimentation and Aggregate Fluctuations: Why getting into a recession is easier than getting out of it. University of British Columbia Department of Economics Discussion Paper: 97/24, September.

[9] Grandmont, J.-M., 1998. Expectations Formation and Stability of Large Socioeconomic Systems. Econometrica 66, 741-781.

[10] Grandmont, J.-M., Laroque, G., 1991. Economic Dynamics with Learning: Some Instability Examples. in Equilibrium Theory and Applications, Proceedings of the Sixth International Symposium in Economic Theory and Econometrics. Barnett, Cornet, D'Aspremont, Gabszeqicz, and Mas-Colell (eds). Cambridge University Press, Cambridge, 1991, chap. 11, pp. 247-273. 
[11] Guse, E. (2003a) Stability Properties for Learning with Heterogeneous Expectations and Multiple Equilibria, University of Helsinki Department of Economics Discussion Paper No 574 / August 27, 2003. ISBN 952-10-1224-2.

[12] Guse, E. (2003b) Learning with Heterogeneous Expectations in an Evolutionary World, Mimeo.

[13] Hamilton, J. 1989. A New Approach to the Economic Analysis of Nonstationary Time Series and the Business Cycle. Econometrica 57, 357-384.

[14] Honkapohja, S., Mitra, K. 2003. Learning Stability in Economics with Heterogeneous Agents. Mimeo.

[15] Kasa, K. (1995) Signal Extraction and the Propagation of Business Cycles. Federal Reserve Bank of San Francisco Working Papers in Applied Economic Theory, 95-14.

[16] Ljungqvist, L. \& T.J. Sargent (2000) Recursive Macroeconomic Theory. The MIT Press, Cambridge.

[17] Moreno, D., Walker, M., 1994. Two Problems in Applying Ljung's "Projection Algorithms" to the Analysis of Decentralized Learning. Journal of Economic Theory 62, 420-427.

[18] Nieuwerburgh, S. \& L. Veldkamp (2002) Learning Asymmetries in Real Business Cycles, Mimeo.

[19] Potter, S. (1999) Fluctuations in Confidence and Asymmetric Business Cycles, Mimeo.

[20] Sargent, T.J. (1999) The Conquest of American Inflation. Princeton University Press, Princeton.

[21] Taylor, J. (1977) Conditions for Unique Solutions in Stochastic Macroeconomic Models with Rational Expectations. Econometrica, 45, 1377-1386.

[22] Timmermann, A., 1996. Excess Volatility and Predictability of Stock Prices in Autoregressive Dividend Models with Learning. The Review of Economic Studies 63, 523-557.

[23] Williams, N. (2003a) Adaptive Learning and Business Cycles, Mimeo. 
[24] Williams, N. (2003b) Escape Dynamics in Learning Models, Mimeo. 